PAPER

\title{
A longitudinal study of sentence comprehension difficulty in primary progressive aphasia
}

\author{
M Grossman, P Moore
}

J Neurol Neurosurg Psychiatry 2005;76:644-649. doi: 10.1136/jnnp.2004.039966

See end of article for authors' affiliations

....................

Correspondence to: Murray Grossman, Department of Neurology-2 Gibson, Hospital of the University of Pennsylvania, 3400 Spruce Street, Philadelphia, PA 191044283, USA; mgrossma@ mail.med.upenn.edu

Received 23 February 2004 Revised version received 4 September 2004 Accepted

7 September 2004
Context: Patients with primary progressive aphasia have sentence comprehension difficulty, but the longitudinal course of this deficit has not been investigated.

Objective: To determine how grammatical, single word meaning, and working memory factors contribute to longitudinal decline of sentence comprehension in primary progressive aphasia. We hypothesised partially distinct patterns of sentence comprehension difficulty in subgroups of patients with progressive non-fluent aphasia (PNFA) and semantic dementia (SD).

Design: Cohort.

Setting: Institutional out patient referral centre.

Patients: PNFA $(n=14)$, SD $(n=10)$.

Main outcome measure: Sentence comprehension accuracy

Results: PNFA patients were significantly impaired at understanding grammatically complex sentences when first seen, and this was more evident than impairment of their comprehension of grammatically simple sentences $(p<0.05)$. Comprehension of grammatically complex sentences correlated with their working memory deficit at presentation $(p<0.05)$. PNFA patients showed modest decline over time in grammatical comprehension. In SD, comprehension of grammatically complex sentences was not more impaired than comprehension of grammatically simple sentences when first seen, but these patients demonstrated a significant longitudinal decline in understanding grammatically complex sentences $(p<0.05)$. Cox regression analyses showed that a deficit in single word meaning contributes to the progressive impairment for grammatically complex sentences in $\operatorname{SD}(p<0.05)$, but working memory does not contribute to longitudinal decline in PNFA.

Conclusion: Patients with PNFA and SD have sentence comprehension difficulty, but distinct factors contribute to this impairment during the course of their disease.
$\mathrm{P}$ atients with primary progressive aphasia have sentence comprehension difficulty. ${ }^{12}$ Clinical observations describe changing speech patterns over time, but longitudinal studies of declining sentence comprehension are found only in informal descriptions of case reports. The nonfluent form of progressive aphasia, known as progressive non-fluent aphasia (PNFA), presents with effortful speech that may be agrammatic or dysarthric. Clinical descriptions of single case studies ${ }^{3-6}$ and small series of PNFA patients ${ }^{7-10}$ note sparser spontaneous speech as the disease progresses, and many patients reportedly become mute. The fluent form of progressive aphasia, also known as semantic dementia (SD), is characterised by fluent speech with frequent word finding pauses and circumlocutions, and difficulty understanding single words and objects. ${ }^{11-13}$ Rare reports describe declining naming and single word comprehension over time. ${ }^{14-17}$ In the present study, we examined the contributions of grammatical processing, single word comprehension, and working memory capacity to declining sentence comprehension in PNFA and SD.

Understanding a sentence is complex. In brief, information represented in the individual content words of a sentence must be determined. This includes both single word meaning and the thematic relationships specified by content words such as who can do what to whom. The long distance, grammatically mediated relationships between words in the sentence also must be established. Sentences emerge over time, so executive resources like working memory are needed to retain words and phrases of a sentence transiently until they can be incorporated into the sentence. Sentence comprehension impairment in PNFA has been documented on measures of sentence-picture matching ${ }^{8}$ and by responses to simple probes of sentences such as who performed the sentence's action. ${ }^{7918}$ These reports have emphasised the role of a grammatical deficit in PNFA patients' sentence comprehension difficulty by showing reduced comprehension accuracy for sentences that feature more complex grammatical structures. On-line studies of sentence comprehension also have been reported that minimise the confounding role of task related resources. In this work, subjects are asked to detect a word in a sentence. Unbeknownst to subjects, the word occasionally follows a grammatical agreement violation. Control subjects demonstrate their sensitivity to grammatical agreements by showing a slight but reliable delay in word detection during the temporal window for processing a grammatical agreement immediately following a grammatical violation relative to their latency to detect a word immediately following a correct grammatical agreement. PNFA patients, by comparison, do not show this kind of delay. One study interpreted this as evidence for their insensitivity to grammatical agreements. ${ }^{19}$ Another study showed that PNFA patients are delayed in word detection following a grammatical agreement violation only when the target word occurs well after the normal processing window. ${ }^{20}$ This pattern of on-line performance correlated with a measure of working memory. The authors suggested that slowed information processing speed allows information to degrade in working memory during the course of grammatical processing. These observations implicate

Abbreviations: PNFA, progressive non-fluent aphasia; SD, semantic dementia 
grammatical and working memory components of sentence processing in the comprehension deficits of PNFA patients.

Studies of SD have documented relatively preserved grammatical processing, ${ }^{821}$ and one on-line assessment of grammatical agreement sensitivity in a small number of SD patients showed a reasonably normal pattern of performance..$^{20}$ Impaired sentence comprehension nevertheless may be seen in these patients, possibly due to the degraded neural representation of the individual content words constituting a sentence. This can interfere with single word meaning and with thematic roles specifying who can do what to whom in a sentence.

While this work suggests a role for single word, grammatical, and working memory limitations in the sentence comprehension difficulties of PNFA and SD, it is unclear how these components conspire to worsen sentence comprehension over time in these patients. The present study was designed to investigate declining sentence comprehension longitudinally in PNFA and SD, and to examine the relative contributions of single word, grammatical, and working memory components to the progressive sentence comprehension deficits observed in these patients.

\section{METHODS}

\section{Subjects}

We studied 24 patients with progressive aphasia who met published criteria for frontotemporal dementia. ${ }^{22}{ }^{23}$ Initial clinical diagnosis was established by an experienced neurologist (MG). This diagnosis was consistent with the results of serum studies, structural imaging studies such as MRI or CT, studies of cerebrospinal fluid (when available), and clinical functional neuroimaging studies such as SPECT or PET. Subsequently, at least two trained reviewers of a consensus committee confirmed the presence of specific diagnostic criteria based on independent reviews of a semi-structured history, detailed mental status exam, and complete neurologic exam (imaging and laboratory studies were not available to the consensus committee). If the reviewers disagreed in their diagnosis ( $18 \%$ of the entire series of 220 cases, including patients with other neurodegenerative diseases), consensus was established through open discussion by the full committee. These patients and their legal representatives participated in an informed consent procedure approved by the Institutional Review Board at the University of Pennsylvania.

Fourteen of these patients had PNFA. PNFA is marked by slow, effortful speech with phonemic paraphasic errors that may be associated with agrammatism or dysarthria. We also examined 10 patients with SD. These patients characteristically have fluent and circumlocutory spontaneous speech with word finding pauses and possibly semantic errors. Subgroup criteria were based on published diagnostic features $^{24}$ modified to improve reliability. ${ }^{25} 26$ We used the consensus mechanism described above to establish subgroup diagnosis. Table 1 summarises the demographic and clinical features of these patients when first enrolled in this study. PNFA and SD patient groups were matched in age $\left(F_{1,22}=0.24 ; \mathrm{NS}\right)$, education $\left(F_{1,22}=1.86 ; \mathrm{NS}\right)$, duration of disease $\left(F_{1,22}=1.98 ; \mathrm{NS}\right)$, and Mini Mental State Exam $\left(\right.$ MMSE) $\operatorname{score}^{27}\left(F_{1,22}=0.23 ; \mathrm{NS}\right)$. The speech characteristics of these patients at first contact are summarised in table 2, and their neuropsychological performance at the initial evaluation is summarised in table 3.

Exclusion criteria included the presence of other neurologic conditions such as stroke or hydrocephalus, primary psychiatric disorders such as depression or psychosis, or a systemic illness that can interfere with cognitive functioning. Most of these patients were taking a fixed dosage of an acetylcholinesterase inhibitor (for example, donepezil,
Table 1 Demographic features of PNFA and SD patients at first contact

\begin{tabular}{lll}
\hline & PNFA $(\mathbf{n}=14)$ & SD $(\mathbf{n}=10)$ \\
\hline Age, years & $64.1(10.3)$ & $66.2(9.7)$ \\
Education, years & $14.4(2.8)$ & $15.9(2.7)$ \\
Duration, months & $29.1(17.9)$ & $39.9(19.5)$ \\
MMSE, max =30 & $23.3(6.2)$ & $24.3(3.1)$ \\
\hline
\end{tabular}

Values in parentheses are standard deviations.

rivastigmine, or galantamine). Some of these patients may have been medicated with a low dosage of a non-sedating anti-depressant (for example, serotonin specific re-uptake inhibitors such as sertraline) or an atypical neuroleptic agent (for example, quetiapine), as indicated clinically, but none of the patients demonstrated any evidence of sedation suggesting over-medication.

\section{Materials and procedure}

We assessed performance on measures of sentence comprehension, single word meaning, and verbal working memory. Tasks were administered amongst other measures during a 45 min session in a quiet room by a trained technician, and were presented in a fixed order (sentence comprehension before working memory and word meaning). This protocol was typically administered on an annual basis, and was repeated for as many sessions as the patient was able to participate. PNFA patients were tested over an average (standard deviation) of 3 (1.3) sessions separated on average by $11(6.2)$ months. SD patients were tested over an average of 3 (1.2) sessions separated on average by 11 (2.8) months. Rates of longitudinal observation did not differ between groups. In the context of a longitudinal course of 4-5 years following diagnosis, ${ }^{28}$ this represents reasonable coverage of the patient's testable lifespan. The cognitive measures included sentence comprehension, verbal working memory, and single word meaning.

\section{Sentence comprehension ${ }^{21}$}

Patients responded to a simple question about the agent or the patient delivered in a brief, aurally presented sentence composed of familiar content words. The sentences had a simple subject-verb-object structure (for example, "The fat old chicken chased the young duck: which bird was chased?") ("simple" sentences; $\mathrm{n}=4$ ) or a subordinate clause structure featuring a subject-relative clause (for example, "The eagle that chased the hawk is fast: which bird was chased?") or an object-relative clause (for example, "The car that the truck hit was green: which vehicle was hit?") ("complex" sentences; $\mathrm{n}=8$ ). Simple and complex sentences were equated for word length without changing grammatical complexity by manipulating the number of filler

Table 2 Percent of PNFA and SD patients with impaired speech characteristics at first contact*

\begin{tabular}{lcl}
\hline & PNFA & SD \\
\hline Dysarthria (\%) & 7.1 & 11.1 \\
Reduced speech rate (\%) & 78.6 & 11.1 \\
Word finding pauses (\%) & 92.9 & 77.8 \\
Circumlocutions (\%) & 42.9 & 66.7 \\
Grammatical errors (\%) & 35.7 & 11.1 \\
Phonemic paraphasic errors (\%) & 57.1 & 22.2 \\
Semantic paraphasic errors (\%) & 28.6 & 33.3 \\
Impaired repetition (\%) & 71.4 & 22.2
\end{tabular}

*The clinical chart of one SD patient was unavailable due to technical reasons. 
Table 3 Mean neuropsychological performance of PNFA and SD patients at first contact

\begin{tabular}{lll}
\hline & PNFA & SD \\
\hline $\begin{array}{l}\text { Episodic memory recognition } \\
\text { accuracy ( } \mathrm{n}=20 \text { ) }\end{array}$ & $85.7 \%(17.4 \%)$ & $79.0 \%(17.2 \%)$ \\
$\begin{array}{l}\text { Boston Naming Test (\% correct, } \\
\mathrm{n}=15)\end{array}$ & $80.0 \%(25.0 \%)$ & $62.7 \%(27.6 \%)$ \\
$\begin{array}{l}\text { Animal naming fluency (no. of } \\
\text { unique correct names/60 s) }\end{array}$ & $10.4(6.2)$ & $7.5(3.8)$ \\
$\begin{array}{l}\text { Letter (F-A-S) naming fluency } \\
\text { (no. of unique correct names/60 s) }\end{array}$ & $5.2(6.4)$ & $9.3(4.7)$ \\
$\begin{array}{l}\text { Digit span forward } \\
\text { Visual constructions accuracy, } \\
\text { max } 11\end{array}$ & $5.2(1.4)$ & $5.1(1.9)$ \\
\hline $\begin{array}{l}\text { *Data were available for this task in } 11 \text { PNFA patients and seven SD } \\
\text { patients. } \\
\text { Values in parentheses are standard deviations. }\end{array}$ & $10.0(1.7)$ \\
\hline
\end{tabular}

adjectives. All sentences were semantically unbiased so that neither noun of a sentence was more likely to be the agent of the named action. Content words were matched for frequency across grammatically simple and complex sentences. Patients heard the sentences twice, presented at a natural cadence with a normal stress pattern. A sentence was repeated at a patient's request.

\section{Verbal working memory ${ }^{29}$}

A sequence of random single digits was presented at a rate of $1 / \mathrm{s}$, and patients repeated the digits in an order reversing the order of presentation. The stimulus sequence began at a length of two digits, and sequence length increased by one digit until a patient failed to repeat two trials of a reversed sequence at a specific length.

\section{Single word meaning 2}

Patients were shown 48 familiar stimuli one at a time and asked to judge whether each was a member of a known target category. One target category was natural (vegetables) and another was manufactured (tools). Half of the stimuli were category exemplars and half were foils. For each category and each stimulus type, half of the items were individual words printed on a $3 \times 5$ inch index card and half were colour photos. Stimuli were matched across categories for frequency and familiarity; the picture stimuli were also matched for visual complexity. The category exemplars and foils were presented in a fixed random order and were otherwise blocked by category and material.

\section{Statistical considerations}

The relative contribution of grammatical, single word meaning, and working memory to sentence comprehension was assessed at first presentation with $t$ tests and Pearson correlations. We used a Cox regression procedure to evaluate the longitudinal sentence comprehension performance of patients. To place the patients' performance in the context of their progressive disease, we used each primary caregiver's determination of the historical point of disease onset, and then divided the patient's clinical history into 6 month segments. Disease onset was then aligned across individual patients, and we placed cognitive probes on this time line prior to analysing performance with a Cox survival analysis. To evaluate the grammatical component of sentence comprehension, we assessed comprehension of grammatically simple sentences separately from grammatically complex sentences. We set a performance end point at 50\% correct (that is, random response to a dichotomous probe). We investigated the role of verbal working memory and individual word meaning in sentence comprehension by dividing the cohorts of patients depending on their verbal working memory performance and their single word meaning performance at the first assessment. The cut off for impaired verbal working memory or impaired word meaning was a $\mathrm{z}$ score less than -1.96 ( significant at the $\mathrm{p}<0.05$ level, two tailed), relative to a group of 25 age and education matched healthy elderly subjects.

\section{RESULTS}

\section{Cross sectional characteristics of sentence} comprehension difficulty

Sentence comprehension accuracy in PNFA and SD patients when first seen is summarised in table 4 . It can be seen that comprehension at first contact was more impaired for the complex sentences than the simple sentences in PNFA, while the simple sentences were more difficult than the complex sentences for the SD patients. Inspection of individual patient performance profiles showed a pattern of worse performance for grammatically complex sentences compared to grammatically simple sentences in eight $(57 \%)$ of the 14 PNFA patients, while two additional patients were equally impaired in their comprehension of grammatically simple and grammatically complex sentences. The pattern of worse performance for grammatically complex sentences compared to grammatically simple sentences was seen only in two $(20 \%)$ of the SD patients $\left(\chi^{2}=4.56 ; \mathrm{p}<0.05\right)$. There was little correlation between comprehension accuracy for simple sentences and complex sentences (PNFA: $r=-0.13$; SD: $r=-0.18$ ) at first contact.

Correlations between sentence comprehension, verbal working memory, and single word meaning when first seen are provided in table 5. We found a significant correlation between comprehension of grammatically complex sentences and verbal working memory performance in PNFA near the onset of the disease process. This correlation was specific for grammatically complex sentences in PNFA, since there were no significant correlations with grammatically simple sentences. None of these correlations were significant in SD when first seen. These findings suggest a deficit in grammatical comprehension early in the course of PNFA that is related in part to the limited working memory of PNFA patients.

Table 4 shows that sentence comprehension accuracy declined in PNFA and SD. We performed paired sample $t$ tests for each type of sentence in each group. We found a significant difference between performance at presentation and at the final assessment only in SD and only for grammatically complex sentences $(\mathrm{t}(9)=2.25$; $\mathrm{p}<0.05)$.

Performance on measures of verbal working memory and single word meaning is also summarised in table 4 . Declines in working memory and single word meaning were present but relatively modest. We used paired sample $t$ tests to evaluate the longitudinal performance of each group on each task by comparing performance at presentation and at the final assessment. We found significant longitudinal decline only in SD and only for single word meaning $(\mathrm{t}(9)=2.58$; $\mathrm{p}<0.05$ ).

\section{Longitudinal studies of sentence comprehension}

Cox regression analyses were used to examine declining sentence comprehension in PNFA and SD. The decline in overall sentence comprehension did not differ between PNFA and SD $\left(\chi^{2}=0.99\right.$; NS). Half of the PNFA patients and half of the SD patients attained their end points of random $(50 \%$ accurate) performance at 3.5 years after disease onset. However, inspection of the slopes of decline suggested a difference between PNFA and SD. Thus, 20\% of PNFA patients attained their end point at 2 years after onset, and $80 \%$ attained their end point at 6 years after disease onset. By 
Table 4 Mean sentence comprehension, working memory, and single word meaning in PNFA and SD patients at first contact and last contact*

\begin{tabular}{|c|c|c|c|c|c|c|c|c|}
\hline & \multicolumn{2}{|c|}{ Sent comp-simple } & \multicolumn{2}{|c|}{ Sent comp-complex } & \multicolumn{2}{|c|}{ Working memory } & \multicolumn{2}{|c|}{ Single word meaning } \\
\hline & First contact & Last contact & First contact & Last contact & First contact & Last contact & First contact & Last contact \\
\hline PNFA & $66.1(27.0)$ & $60.7(21.3)$ & 60.7 (18.9) & $54.5(24.8)$ & $3.3(1.2)$ & $2.2(1.7)$ & $83.7(11.6)$ & $81.2(15.7)$ \\
\hline SD & $57.5(23.7)$ & $50.0(26.4)$ & $71.3(25.0)$ & $56.3(25.9) \dagger$ & $3.4(2.2)$ & $3.1(1.7)$ & $84.3(11.5)$ & $75.4(17.5) \dagger$ \\
\hline
\end{tabular}

*Sentence comprehension (Sent comp) and single word meaning measures are \% correct, and working memory measure is the number of digits repeated correctly in the reverse order; tsignificant differences ( $p<0.05$, according to $t$ tests) between first contact and last contact were found in SD for comprehension of grammatically complex sentences and single word meaning.

Values in parentheses are standard deviations.

comparison, SD patients showed a steeper decline, with none of the patients having attained their end point at 2 years after disease onset, but all SD patients having attained their end point at 6 years after disease onset.

We examined the basis for declining sentence comprehension in greater detail by dividing each cohort into subgroups depending on performance when first seen. To assess the role of single word meaning, we subgrouped patients into those with relatively intact single word meaning at presentation compared to patients with relatively impaired single word meaning at presentation. A Cox regression analysis of these subgroups is illustrated in fig 1. Analyses revealed that SD patients with impaired single word meaning have a more rapid decline in the comprehension of grammatically complex sentences compared to SD patients with relatively preserved single word meaning $\left(\chi^{2}=5.48 ; \mathrm{p}<0.02\right)$. There was no difference between SD subgroups for simple sentences $\left(\chi^{2}=1.88\right.$; NS). These findings demonstrate the selective impact of declining single word representations on grammatically complex sentence comprehension in SD. In PNFA, single word meaning had no impact on the comprehension of grammatically simple sentences $\left(\chi^{2}=0.01 ; \quad\right.$ NS $)$ or grammatically complex sentences $\left(\chi^{2}=0.28 ; \mathrm{NS}\right)$. These observations suggest that impaired single word meaning contributes to declining sentence comprehension in SD.

We examined the role of verbal working memory in declining sentence comprehension using a similar approach. Thus, we separated PNFA and SD cohorts into subgroups with relatively intact verbal working memory at presentation and patients with relatively impaired verbal working memory at presentation. A Cox regression analysis of sentence comprehension in these subgroups is illustrated in fig 2 . We did not find a difference for complex grammatical comprehension between subgroups of PNFA patients with impaired versus intact working memory $\left(\chi^{2}=0.03\right.$; NS $)$. We also failed to find evidence that the integrity of working memory had an impact on the comprehension of grammatically simple sentences in PNFA $\left(\chi^{2}=0.13\right.$; NS). In SD, we found considerable decline in the comprehension of grammatically complex sentences in patients with impaired working memory, but there were too few SD patients with intact working memory to assess relative decline statistically.

Table 5 Correlations between sentence comprehension accuracy and performance on measures of working memory and single word meaning in PNFA and SD

\begin{tabular}{llll}
\hline & $\begin{array}{l}\text { Sentence } \\
\text { comprehension }\end{array}$ & $\begin{array}{l}\text { Verbal working } \\
\text { memory }\end{array}$ & $\begin{array}{l}\text { Semantic } \\
\text { memory }\end{array}$ \\
\hline PNFA & Simple & -0.09 & 0.01 \\
SD & Complex & $0.53^{*}$ & 0.50 \\
& Simple & 0.04 & 0.10 \\
& Complex & 0.31 & 0.30 \\
\hline
\end{tabular}

${ }^{*}$ This correlation is significant at the $p<0.05$ level.
The association between limited working memory and impaired grammatical comprehension at the onset of PNFA does not apparently lead to additional comprehension difficulty in these patients over time.

\section{DISCUSSION}

Our observations provide the first empirical evidence that sentence comprehension declines over time in patients with primary progressive aphasia. ${ }^{7-9}$ We found that patients with PNFA are quite impaired in their sentence comprehension when first seen, particularly their comprehension of grammatically complex sentences. A correlation analysis associated their grammatical comprehension deficit at presentation with limited working memory. PNFA patients' sentence comprehension declined modestly over time, probably because the patients were so impaired when first seen that they did not differ from random performance. SD patients' sentence comprehension did change over time. SD patients with impaired single word meaning declined more significantly in their comprehension of grammatically complex sentences than SD patients with relatively preserved single word meaning. We argue below that degraded single word representations and impaired working memory contribute to distinct patterns of declining sentence comprehension in PNFA and SD.

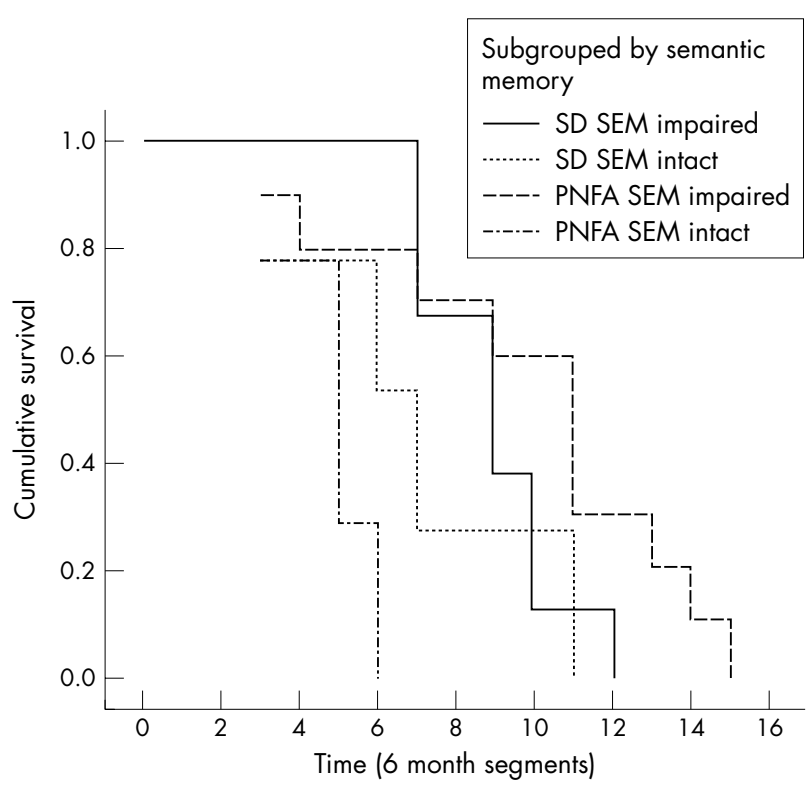

Figure 1 Comprehension of grammatically complex sentences in patients with PNFA and SD subgrouped according to their single word meaning: survival function at mean of covariates. End point for sentence comprehension set at $50 \%$ accuracy. Single word meaning (SEM) is considered impaired at a $z$ score of less than $-1.96(p<0.05$, corrected) relative to 25 age and education matched controls. 


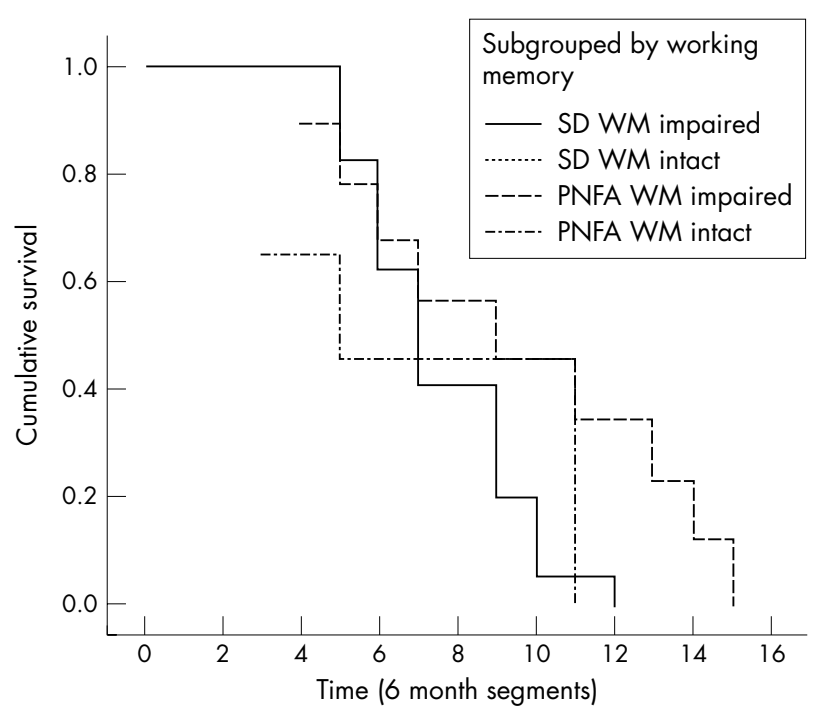

Figure 2 Comprehension of grammatically complex sentences in patients with PNFA and SD subgrouped according to their working memory: survival function at mean of covariates. End point for sentence comprehension set at $50 \%$ accuracy. Working memory is considered impaired at a $z$ score of less than -1.96 ( $p<0.05$, two tailed) relative to 25 age and education matched controls. There were too few SD patients with intact working memory to plot longitudinal performance for grammatically complex sentences.

Early in the course of progressive aphasia, patients with PNFA have greater difficulty understanding grammatically complex sentences compared to grammatically simple sentences. This effect for grammatical complexity has been observed in other assessments of sentence comprehension in PNFA. $^{71920}$ One factor contributing to the deficit with grammatically complex sentences early in the course of PNFA appears to be verbal working memory. Previous work suggested that verbal working memory contributes to comprehension of grammatical features such as interpreting subordinate clauses in sentences, ${ }^{30}$ characteristic of the grammatically complex sentences used in the present study. For example, working memory may be necessary to retain a noun phrase mentally when encountered early in a sentence until it can be linked to a subordinate clause that occurs later in the sentence. ${ }^{3132}$ We have shown that this working memory deficit is related to the comprehension of sentences rather than task related resources in a study of on-line sentence processing in PNFA. ${ }^{20}$

We did not observe a significant decline in comprehension accuracy over time in PNFA. These patients may not have been able to decline much further statistically since their performance with grammatically complex sentences did not differ from random at presentation. This may be related in part to the neuroanatomic distribution of disease in PNFA. Studies examining cortical atrophy and limited cortical metabolism have associated PNFA with disease in the left inferior frontal region, ${ }^{73-35}$ and a correlation study associated difficulty understanding grammatically complex sentences with a voxel based morphometric analysis of left inferior frontal cortical volume in PNFA. ${ }^{18}$ A BOLD fMRI study of sentence comprehension in healthy elderly subjects showed left inferior frontal activation during the comprehension of grammatically complex sentences, ${ }^{36}$ but PNFA patients with grammatical comprehension difficulty showed only limited left inferior frontal recruitment during their performance with the same sentences. ${ }^{37}$ A portion of the verbal working memory neural network is represented in a frontal region adjacent to this grammatical area, ${ }^{38}$ and recent work has demonstrated the contribution of this area to the processing of grammatically complex sentences. ${ }^{36}{ }^{39}$ Cortical atrophy in this area appears to be related to impaired working memory in PNFA. ${ }^{18}$ These observations are consistent with the hypothesis that the neuroanatomic distribution of disease early in the course of PNFA compromises brain regions crucial to grammatical aspects of sentence comprehension and the working memory support contributing to the processing of these sentences.

We found a different longitudinal pattern of sentence comprehension decline in SD. This difference cannot be explained by non-specific factors such as overall disease severity or disease duration since PNFA and SD patients were matched in these regards. Progressive sentence comprehension decline in SD instead appears to be influenced by a lexical semantic deficit that contributes to sentence processing. Specifically, SD patients with impaired single word comprehension have a steeper decline in their performance with grammatically complex sentences than SD patients with relatively preserved single word comprehension. Yet, single word meaning did not correlate directly with sentence comprehension difficulty. These findings suggest that another facet of a word's representation contributes to the sentence comprehension deficit in SD. Thematic role information is also represented in single words. This helps constrain sentence interpretation by specifying who can do what to whom, and this may be particularly valuable in the comprehension of sentences featuring grammatically complex long distance dependencies. ${ }^{40}$ Single word meaning correlates with left posterolateral and ventral temporal atrophy in SD. ${ }^{41}{ }^{42}$ We are aware of only one report examining the neural correlate of sentence comprehension difficulty in SD. ${ }^{18}$ This study showed a correlation between sentence comprehension accuracy and cortical volume in the posterolateral portion of left temporal cortex. Additional work is needed to determine more directly whether thematic role knowledge correlates with left temporal atrophy in SD.

We cannot rule out that working memory also contributes to sentence comprehension difficulty in SD. Considerable decline was observed in SD patients with impaired working memory, although our sample was too small to examine this empirically. Working memory itself is a multi-component process that is thought to include a limited capacity, limited duration storage device for verbal material, and processes for maintaining this information in short term memory such as a phonological rehearsal loop. ${ }^{43}$ Functional neuroimaging studies associate a storage component of verbal working memory with left inferior parietal cortex. ${ }^{44}$ While this region may not be compromised in SD, other patients with a fluent form of progressive aphasia may have disease in this distribution that contributes to their sentence comprehension difficulty. ${ }^{13}$

Regardless of the basis for sentence comprehension difficulty in these patients, the different patterns of impaired sentence processing in PNFA and SD suggest that there are at least two distinct subgroups of patients with progressive aphasia. ${ }^{1}$ In contrast to clinical descriptions suggesting that progressive aphasia is a single, undifferentiated syndrome, ${ }^{23}$ our findings are more consistent with comparative empirical observations $^{82045}$ and quantitative structural imaging studies $^{13}{ }^{41}$ suggesting that PNFA and SD represent distinct subgroups of progressive aphasia.

Several caveats should be kept in mind when interpreting our findings. It would be important to determine whether other grammatical features of sentence structure are equally difficult for PNFA patients. The relatively modest number of follow up studies for each individual, and the small number of patients contributing to each subgroup, suggest caution in the interpretation of the Cox regression analyses. Our 
findings, nevertheless, suggest that sentence comprehension declines over time in primary progressive aphasia. The precise longitudinal course of this difficulty appears to vary depending on several interacting factors. Among these are the status of grammatical processing, the integrity of the single word representations, and the condition of working memory in each subgroup of progressive aphasia. PNFA patients appear to have a grammatical comprehension deficit near the beginning of their disease, related in part to their early working memory impairment. Grammatical comprehension declines over time in SD, by comparison, apparently related to progressive difficulty with single word representation.

\section{Authors' affiliations}

M Grossman, P Moore, Department of Neurology, University of Pennsylvania, Philadelphia, PA, USA

Portions of this work were supported by the US Public Health Service (AG17586, AG15116, and NS44266)

Competing interests: none declared

\section{REFERENCES}

1 Grossman M, Ash S. Primary progressive aphasia: a review. Neurocase 2004; 10:3-18.

2 Mesulam M-M. Primary progressive aphasia. Ann Neurol 2001 ;49:425-32

3 Kertesz A, Hudson L, Mackenzie IR, et al. The pathology and nosology of primary progressive aphasia. Neurology 1994:44:2065-72.

4 Mesulam M-M. Slowly progressive aphasia without generalized dementia. Ann Neurol 1982;11:592-8.

5 Snowden JS, Neary D, Mann DMA, et al. Progressive language disorder due to lobar atrophy. Ann Neurol 1992;31:174-83.

6 Snowden JS, Neary D. Progressive language dysfunction and lobar atrophy. Dementia 1994:4:226-31.

7 Grossman M, Mickanin J, Onishi K, et al. Progressive non-fluent aphasia: language, cognitive and PET measures contrasted with probable Alzheimer's disease. J Cogn Neurosci 1996;8:135-54.

8 Hodges JR, Patterson K. Nonfluent progressive aphasia and semantic dementia: a comparative neuropsychological study. J Int Neuropsychol Soc 1996;2:51 1-24.

9 Thompson CK, Ballard KJ, Tait ME, et al. Patterns of language decline in nonfluent primary progressive aphasia. Aphasiology 1997; 11:297-331.

10 Weintraub S, Rubin NP, Mesulam M-M. Primary progressive aphasia: longitudinal course, neuropsychological profile, and language features. Arch Neurol 1990;47:1329-35.

11 Hodges JR, Patterson K, Oxbury S, et al. Semantic dementia: progressive fluent aphasia with temporal lobe atrophy. Brain 1992;115:1783-806.

12 Snowden JS, Goulding PJ, Neary D. Semantic dementia: a form of circumscribed cerebral atrophy. Behav Neurol 1989;2:167-82.

13 Gorno-Tempini M, Dronkers NF, Rankin KP, et al. Cognition and anatomy in three variants of primary progressive aphasia. Ann Neurol 2004;55:335-46.

14 Barbarotto R, Capitani E, Spinnler H, et al. Slowly progressive semantic impairment with category specificity. Neurocase 1995;1:107-19.

15 Basso A, Capitani E, Laiacona M. Progressive language impairment without dementia: a case with isolated category-specific semantic impairment. J Neurol Neurosurg Psychiatry 1988;51:1201-7.

16 Diesfeldt HFA. Progressive decline of semantic memory with preservation of number processing and calculation. Behav Neurol 1993;6:239-42.

17 Hodges JR, Patterson KE, Tyler LK. Loss of semantic memory: implications for the modularity of mind. Cogn Neuropsychol 1994;11:505-42.

18 Grossman M, Work M, Gee JC, et al. Sentence comprehension difficulty in progressive non-fluent aphasia: a voxel-based morphometric analysis (submitted for publication).
19 Tyler LK, Moss HE, Patterson K, et al. The gradual deterioration of syntax and semantics in a patient with progressive aphasia. Brain Lang 1997;56:426-76

20 Grossman M, Rhee J, Antiquena P. Sentence processing in frontotemporal dementia. Cortex (in press).

21 Grossman $M, D^{\prime}$ Esposito $M$, Hughes $E$, et al. Language comprehension difficulty in Alzheimer's disease, vascular dementia, and fronto-temporal degeneration. Neurology 1996:47:183-9.

22 The Lund and Manchester Groups. Clinical and neuropathological criteria for frontotemporal dementia. J Neurol Neurosurg Psychiatry 1994;57:416-8.

23 McKhann G, Trojanowski JQ, Grossman M, et al. Clinical and pathological diagnosis of frontotemporal dementia: report of a work group on frontotemporal dementia and Pick's disease. Arch Neurol 2001:58:1803-9.

24 Neary D, Snowden JS, Gustafson L, et al. Frontotemporal lobar degeneration: a consensus on clinical diagnostic criteria. Neurology 1998;51:1546-54.

25 Davis KL, Price C, Moore P, et al. Evaluating the clinical diagnosis of frontotemporal degeneration: a re-examination of Neary et al, 1998. Neurology 2001;56:A144-5.

26 Price C, Davis KL, Moore $\mathrm{P}$, et al. Clinical diagnosis of frontotemporal dementia (FTD). Neurology 2001;56:A176.

27 Folstein MF, Folstein SE, McHugh PR. "Mini-mental state." A practical method for grading the cognitive state of patients for the clinician. J Psychiatr Res 1975; 12:189-98

28 Hodges JR, Davies R, Xuereb J, et al. Survival in frontotemporal dementia. Neurology 2003:61:349-54.

29 Wechsler D. Wechsler adult intelligence scale. San Antonio, TX: Psychological Press, 1995.

30 Caplan D, Waters GS. Verbal working memory and sentence comprehension. Behav Brain Sci 1999;22:77-126.

31 Swinney D, Zurif EB, Prather $P$, et al. Neurological distribution of processing resources underlying language comprehension. J Cogn Neurosci 1996;8:174-84.

32 Zurif EB, Swinney D, Prather $P$, et al. The allocation of memory resources during sentence comprehension: evidence from the elderly. J Psycholinguistic Res 1995;24:165-82.

33 Chawluk J, Mesulam M-M, Hurtig HI, et al. Slowly progressive aphasia without dementia: studies with positron emission tomography. Ann Neurol 1986;19:68-74.

34 Kempler D, Metter EJ, Riege WH, et al. Slowly progressive aphasia: three cases with language, memory, CT, and PET data. J Neurol Neurosurg Psychiatry 1990;53:987-93.

35 Nestor PJ, Graham NL, Fryer TD, et al. Progressive non-fluent aphasia is associated with hypometabolism centred on the left anterior insula. Brain 2003; 126:2406-18

36 Grossman M, Cooke A, DeVita C, et al. Age-related changes in working memory during sentence comprehension: an $\mathrm{FMRI}$ study. Neuroimage 2002; 15:302-17.

37 Cooke A, DeVita C, Gee JC, et al. Neural basis for sentence comprehension deficits in frontotemporal dementia. Brain Lang 2003;85:211-21.

38 Smith EE, Jonides J. Storage and executive processes in the frontal lobes. Science 1999;283:1657-61.

39 Cooke A, DeVita C, Gonzalez-Atavales J, et al. Large-scale neural network for sentence processing (submitted for publication).

40 Grimshaw J. Argument structure. Cambridge, MA: MIT Press, 1990.

41 Grossman M, McMillan C, Moore P, et al. What's in a name: voxel-based morphometric analyses of MRI and naming difficulty in Alzheimer's disease, frontotemporal dementia, and corticobasal degeneration. Brain 2004; 127:628-49.

42 Mummery CJ, Patterson K, Price CJ, et al. A voxel-based morphometry study of semantic dementia: relationship between temporal lobe atrophy and semantic memory. Ann Neurol 2000;47:36-45.

43 Baddeley AD. Working memory, 1 st ed. Oxford: Clarendon Press, 1986.

44 Smith EE, Jonides J, Marshuetz C, et al. Components of verbal working memory: evidence from neuroimaging. Proc Natl Acad Sci U S A 1998;95:876-82

45 Rhee J, Moore P, Grossman M. Verb comprehension in frontotemporal degeneration: the role of grammatical, semantic and executive components. Neurocase $2001 ; 7: 173-84$ 